\title{
Fast Near-Field Calculation for Volume Integral Equations for Layered Media
}

\author{
Kim, Oleksiy S.; Meincke, Peter; Breinbjerg, Olav
}

Published in:

5th International Conference on Antenna Theory and Techniques

Link to article, DOI:

10.1109/ICATT.2005.1497004

Publication date:

2005

Document Version

Publisher's PDF, also known as Version of record

Link back to DTU Orbit

Citation (APA):

Kim, O. S., Meincke, P., \& Breinbjerg, O. (2005). Fast Near-Field Calculation for Volume Integral Equations for Layered Media. In 5th International Conference on Antenna Theory and Techniques (pp. 427-429). IEEE. https://doi.org/10.1109/ICATT.2005.1497004

\section{General rights}

Copyright and moral rights for the publications made accessible in the public portal are retained by the authors and/or other copyright owners and it is a condition of accessing publications that users recognise and abide by the legal requirements associated with these rights.

- Users may download and print one copy of any publication from the public portal for the purpose of private study or research.

- You may not further distribute the material or use it for any profit-making activity or commercial gain

- You may freely distribute the URL identifying the publication in the public portal 


\title{
FAST NEAR-FIELD CALCULATION FOR VOLUME INTEGRAL EQUATIONS FOR LAYERED MEDIA
}

\author{
Oleksiy S. Kim, Peter Meincke, and Olav Breinbjerg
}

\author{
Ørsted-DTU, Electromagnetic Systems, Technical University of Denmark \\ Ørsteds Plads, Building 348, DK-2800 Kgs. Lyngby, Denmark (osk@oersted.dtu.dk)
}

\begin{abstract}
An efficient technique based on the Fast Fourier Transform (FFT) for calculating near-field scattering by dielectric objects in layered media is presented. A higher order method of moments technique is employed to solve the volume integral equation for the unknown induced volume current density. Afterwards, the scattered electric field can be easily computed at a regular rectangular grid on any horizontal plane using a 2-dimensional FFT. This approach provides significant speedup in the nearfield calculation in comparison to a straightforward numerical evaluation of the radiation integral since it eliminates the very time-consuming computations of the dyadic Green's functions for layered media.
\end{abstract}

Keywords: volume integral equation, electromagnetic scattering, higher-order hierarchical basis functions, method of moments, FFT, nearfield.

\section{INTRODUCTION}

When the forward solution of the scattering problem is employed in inversion schemes, the scattered field is often required to be calculated on a regular horizontal rectangular grid with a vast number of nodes. In the presence of layered media this task becomes extremely time-consuming since a straightforward numerical evaluation of the radiation integral involves computations of the multilayer dyadic Green's functions. These computations can be significantly simplified if the distance between the observation point and the scattering object is large enough and consequently, the far-field asymptotic approximation can be utilized. Otherwise, the multilayer Green's functions must be computed exactly for each pair of source and observation points, which takes a great amount of time even if fast methods such as the Discrete Complex Image Method (DCIM) or the model based parameter estimation technique are utilized.

Fortunately, the near-field computations on a rectangular grid can be extensively accelerated by application of the Fast Fourier Transform (FFT). This approach allows the nearfield to be computed simultaneously at all discrete values of $x$ and $y$ for a fixed value of $z$. Moreover, this representation has a transformation kernel in a simple closed form, which eliminates time-consuming calculations of the multilayer Green's functions.

In this paper the fast FFT near-field calculation is combined with the efficient higher order Method of Moments (MoM) solution for volume integral equations (VIE) for layered media [1] that is based on higher order hierarchical Legendre basis functions [2] and higher order geometry modeling.

Numerical examples for buried dielectric objects are presented to validate the fast FFT near-freld calculation technique.

Throughout the paper the time factor $e^{j a x}$ is assumed and suppressed.

\section{FFT NEAR-Field CALCULATION FOR Higher-order Hierarchical MoM}

In a layered medium the scattered electric field at the observation point $(x, y, z)$ in the $i-t h$ layer due to the induced volume current density $\bar{J}(\bar{r})$ immersed in the $n$-th layer can be expanded in terms of plane waves as [3]

$$
\begin{array}{r}
\bar{E}^{s}(x, y, z)=-\frac{\omega \mu_{0}}{8 \pi^{2}} \int_{-\infty-\infty}^{\infty} \int_{0}^{\infty}\left[\bar{F}_{i n}^{ \pm}\left(k_{x}, k_{y}\right) e^{\mp j k_{z} z^{2}}\right] \\
\cdot e^{-j \mid k_{x} x+k_{y} y} d k_{x} d k_{y},
\end{array}
$$

where

$$
\bar{F}_{\text {in }}^{ \pm}\left(k_{x}, k_{y}\right)=\overline{\bar{F}}_{\text {in }}^{ \pm}\left(k_{x}, k_{y}\right) \cdot \tilde{J}^{ \pm}\left(k_{x}, k_{y}\right),
$$

$k_{z i}^{2}=k_{i}^{2}-k_{x}^{2}-k_{y}^{2}, k_{i}$ is a propagation constant in the $i$-th layer, and $\tilde{\bar{J}}^{\ddagger}\left(k_{x}, k_{y}\right)$ is a 3-dimensional (3D) spatial Fourier transform of $\bar{J}(\bar{r})$ known from the solution of the VIE

$$
\tilde{\bar{J}}^{ \pm}\left(k_{x}, k_{y}\right)=\int_{V} \bar{J}\left(\bar{r}^{\prime}\right) e^{j \overline{j i} \cdot \bar{r}^{\prime}} d v^{\prime}
$$

where $\bar{k}=\hat{x} k_{x}+\hat{y} k_{y}+\hat{z} k_{z}$. 
Plus and minus in the expressions correspond to upward and downward propagating waves, respectively. The dyadic $\bar{F}_{\text {in }}^{ \pm}\left(k_{x}, k_{y}\right)$ for a layered medium can be represented in closed form. For instance, in a case of a two-layer medium (Fig. 1) the dyadic is

$\overline{\bar{F}}_{12}^{+}\left(k_{x}, k_{y}\right)=A\left[\begin{array}{ccc}k_{y}^{2}+k_{z 1} k_{z 2} & -k_{x} k_{y} & -k_{x} k_{z 1} \\ -k_{x} k_{y} & k_{x}^{2}+k_{z 1} k_{z 2} & -k_{y} k_{z 1} \\ -k_{x} k_{z 2} & -k_{y} k_{z 2} & k_{x}^{2}+k_{y}^{2}\end{array}\right],(3)$

where $A=\left(k_{z 1}+k_{z 2}\right)^{-1}\left(k_{x}^{2}+k_{y}^{2}+k_{z 1} k_{z 2}\right)^{-1}$, corresponding to an upward propagating wave in layer 1 (upper layer) and a source in layer 2 (lower layer).

The near-field calculation technique used here is efficient due to the 2-dimensional (2-D) FFT employed for evaluation of the integral (1) [4], which allows the field to be computed simultaneously for all discrete values of $x$ and $y$ in a rectangular grid at a $z=$ const plane.

The integrand in (1) is band limited due to the factor $e^{\mp j k_{z^{z}}}$. Hence, the required bandwidth $k_{\max }$ is determined by requiring $e^{-\sqrt{k_{\max }^{2}-k_{i}^{2}}|z|}$ being sufficiently small. The integrations over $k_{x}, k_{y}$ in (1) is then performed in the ranges $-k_{\max }<k_{x}<k_{\max }$, $-k_{\max }<k_{y}<k_{\max }$. Consequently, the grid, on which the scattered fieid (1) is calculated using the FFT, has the spacing $\quad \Delta x=\Delta y=\pi /\left(2 k_{\max }\right)$.

Further acceleration of the near-field computations can be achieved by speeding up the calculation of the 3-D spatial Fourier transform (2) of the induced volume current density $\bar{J}(\bar{r})$. Since the Fourier spectrum of $\bar{J}(\bar{r})$ is not band limited, it is problematic to employ the FFT for evaluation of (2). However, the efficiency can still be improved in an alternative semianalytical way as explained below.

In our Method of Moments solution for volume integral equations [5] a volumetric dielectric object is represented by trilinear and/or curved hexahedral elements, and the volume current density $\bar{J}(\bar{r})$ is defined in a local curvilinear $(u, v, w)$-coordinate system of each element as

$$
\begin{array}{r}
J^{\xi}=\frac{j \omega\left(1-\frac{\varepsilon}{\varepsilon_{b}}\right)}{\mathfrak{I}} \sum_{m=0}^{M^{\xi}} \sum_{n=0}^{N^{\eta}} \sum_{q=0}^{Q^{\zeta}} a_{m i q}^{\xi} \tilde{P}_{m}(\xi) P_{n}(\eta) P_{q}(\zeta) ; \\
(\xi, \eta, \zeta) \text { is }(u, v, w),(v, w, u), \text { or }(w, u, v), \\
-1 \leq u, v, w \leq 1,
\end{array}
$$

where $\varepsilon$ is the relative dielectric permittivity of the element, which generally can be a function of $(u, v, w)$, and $\varepsilon_{b}$ is the relative dielectric permittivity of the background medium. Moreover, $\mathfrak{\Im}$ denotes the Jacobian of the parametric transformation, $P_{m}(\xi)$ are Legendre polynomials, $a_{m n q}^{\xi}$ are coefficients deter- mined in the MoM solution of VIE, and $M^{\xi}, N^{\eta}$, and $Q^{5}$ are current expansion orders. The functions $\tilde{P}_{m}(\xi)$ are defined in [2].

To accelerate the Fourier transform of $\bar{J}(\bar{r})$, an auxiliary 3-dimensional rectangular grid of the size $N_{x}^{\prime} \times N_{y}^{\prime} \times N_{z}^{\prime}$ is defined so that it completely encloses the object, as shown in Fig. 1. Then, each component of the volume current density $\bar{J}(\bar{r})$ is approximated by a series of pulse function as

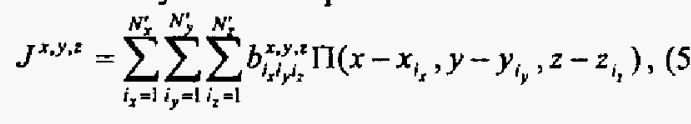

where

$$
\Pi(x, y, z)=\left\{\begin{array}{c}
-\frac{\Delta x^{\prime}}{2}<x<\frac{\Delta x^{\prime}}{2} \\
1,-\frac{\Delta y^{\prime}}{2}<y<\frac{\Delta y^{\prime}}{2} \\
-\frac{\Delta z^{\prime}}{2}<z<\frac{\Delta z^{\prime}}{2} \\
0, \quad \text { elsewhere }
\end{array} .\right.
$$

The coefficients $b_{i_{r} j_{j} i_{s}}^{x, y, z}$ are determined from (4) for each rectangular cell in:ide the object and set to zero elsewhere.

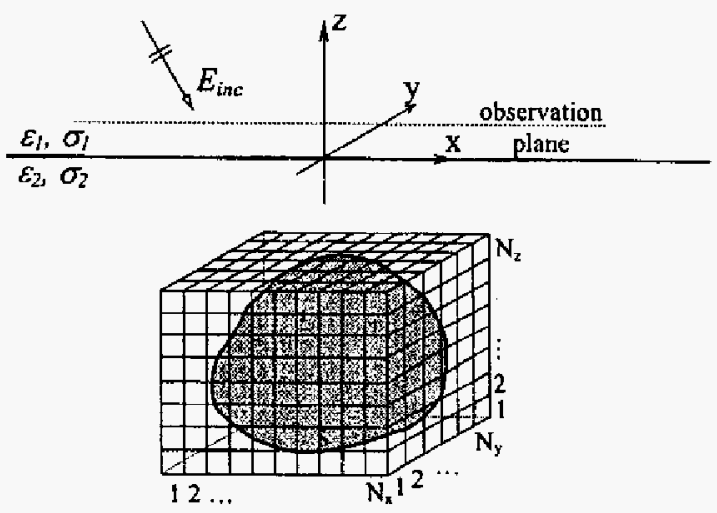

Fig. 1. Dielectric object in a two-layer medium. Definition of the auxiliary grid.

Substituting (5) into (2), and using the fact that the Fourier transform of the pulse function is known in closed form, we can find

$$
\begin{aligned}
& \tilde{\bar{J}}\left(k_{x}, k_{y}\right)=\operatorname{sinc}\left(k_{x} \frac{\Delta x^{\prime}}{2}\right) \operatorname{sinc}\left(k_{y} \frac{\Delta y^{\prime}}{2}\right) \operatorname{sind}\left(k_{z} \frac{\Delta z^{\prime}}{2}\right) \\
& \cdot e^{j k_{x} x_{1}} e^{j k_{y} y_{1}} e^{j k_{z} z_{1}} \Delta x^{\prime} \Delta y^{\prime} \Delta z^{\prime}
\end{aligned}
$$

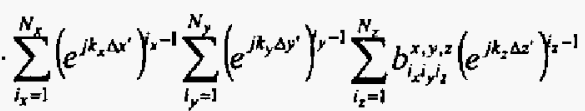

provided that the object is confined to one layer. Thus, the time-consuming calculations of the exponential functions of the complex argument are reduced to the minimum, and the 3-D spatial Fourier 
transform of $\bar{J}(\bar{r})$ can be evaluated in a very efficient manner.

It is worth noting that the kemel $\overline{\bar{F}}_{\text {in }}^{ \pm}\left(k_{x}, k_{y}\right)$ in (1) needs to be computed only once. Then the field at different $z=$ const planes can be calculated simply by a series of 2-D FFT's with the appropriate factor $e^{\mp j k_{x / 2}}$ in (1).

\section{NUMERICAL EXAMPLES}

Consider a two-layer media, in which the upper halfspace is air, and the lower half-space is lossy with the relative permittivity $\varepsilon_{2}=3.0-j 0.1$. A low-contrast homogeneous dielectric cube of the size $0.2 \times 0.2 \times 0.2 \lambda_{0}$ with the relative permittivity $\varepsilon=2.9-j 0.05$ is buried, so that its center is located $0.2 \lambda_{0}$ below the interface. The $y$-polarized plane wave is normally incident from above. Eight cubic cells and the current expansion order $M^{5}=2$ are used in the MoM solution. The scattered electric field along the line $y=0$ for various heights above the interface is computed both by a straightforward numerical integration of the radiation integral and by the presented FFT technique. The results plotted in Fig. 2 demonstrate an excellent agreement between these two methods.

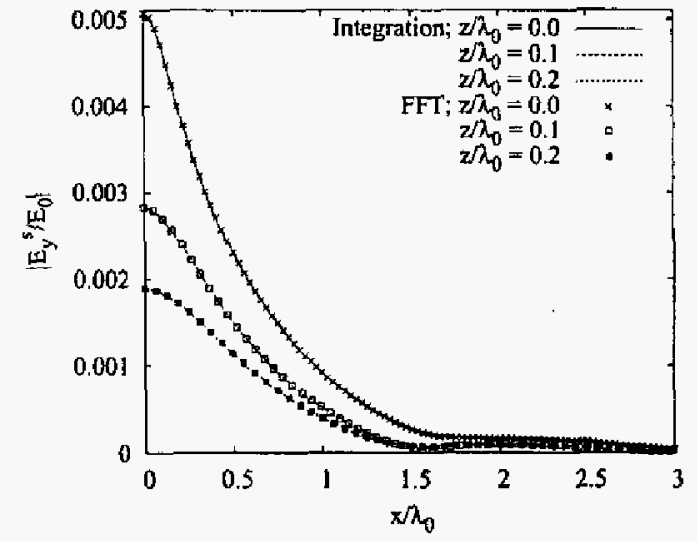

Fig. 2. Scattered nearfield for a cube.

In the next example a buried dielectric slab with higher contrast to the background medium is considered. The relative permittivities of the object and the lower layer are $\varepsilon=10.0-j 5.0$ and $\varepsilon_{2}=4.0-j 0.5$, respectively. The slab has dimensions $0.3 \times 0.3 \times 0.05 \lambda_{0}$, and its bottom face is $0.4 \lambda_{0}$ below the interface. In the MoM solution the object is represented by 4 cells with the current expansion orders $M^{x, y}=3$ and $M^{z}=1$ in the horizontal and vertical directions, respectively. Fig. 3 . shows the scattered nearfield computed by the numerical integration and by the presented FFT technique. Again, a perfect agreement between two methods is observed.

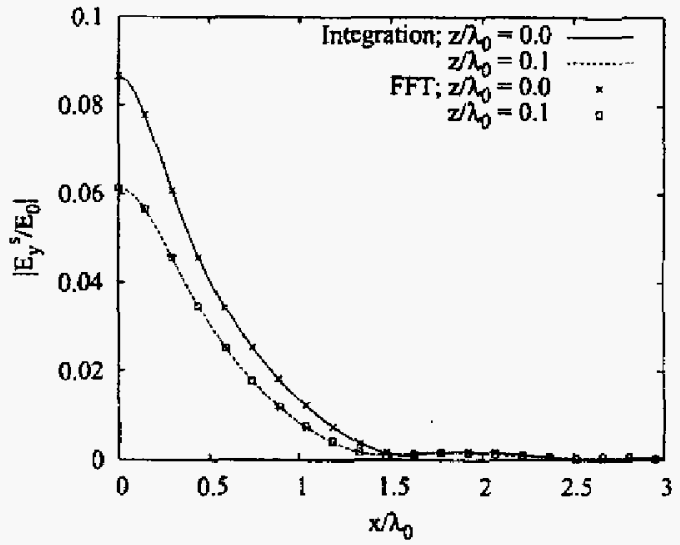

Fig. 3. Scattered nearfield for a slab.

\section{Conclusion}

The fast FFT near-field calculation technique is combined with the higher order MoM for VIE in layered media. The FFT provides an efficient computation of the near-field scattering by dielectric objects in layered media when the observation points are regularly distributed on horizontal rectangular grids. The application of the plane wave expansion method allows the time-consuming computations of the multilayer dyadic Green's functions to be eliminated from the nearfield calculation. An efficient semi-analytical technique is utilized for evaluation of the 3-D Fourier transform of the induced volume current density.

\section{REFERENCES}

1. Kim O.S., Meincke P., Breinbjerg O., "Analysis of Buried Dielectric Objects Using Higher-Order MoM for Volume Integral Equations," Int. Conf. Mathem. Methods in Electromagnetic Theory, Ukraine, September 14-17, pp. 254-256, 2004.

2. Jørgensen E., Volakis J. L., Meincke P., and Breinbjerg $O$., "Higher order hierarchical Legendre basis functions for electromagnetic modeling," IEEE Trans. on Antennas and Propagation, vol. 52, no. 11, pp. 2985-2995, November 2004.

3. Meincke $P$., "Linear GPR inversion for lossy soil and a planar air-soil interface," IEEE Trans. on Geoscience and Remote Sensing, vol. 39, no. 12, pp. 2713-2721, December 2001.

4. Howard A.Q., Jr, "On Approximating Fourier Integral Transforms by Their Discrete Counterparts in Certain Geophysical Application," IEEE Trans. on Antennas and Propagation, vol. 23, pp. 264 266, March 1975.

5. Kim O.S., Jørgensen E., Meincke P., Breinbjerg O., "Method of moments solution of volume integral equations using higher-order hierarchical Legendre basis functions," Radio Science, vol. 39, 2004, RS5003, doi:10.1029/2004RS003041. 